Rabaska

Revue d'ethnologie de l'Amérique française

\title{
Les archives de Marius Barbeau : une richesse à découvrir ou à redécouvrir
}

\section{Benoît Thériault}

Volume 13, 2015

Présence de Marius Barbeau : l'invention du terrain en Amérique

française. Autour d'un legs centenaire (1914-2014)

URI : https://id.erudit.org/iderudit/1033764ar

DOI : https://doi.org/10.7202/1033764ar

Aller au sommaire du numéro

Éditeur(s)

Société québécoise d'ethnologie

ISSN

1703-7433 (imprimé)

1916-7350 (numérique)

Découvrir la revue

Citer cet article

Thériault, B. (2015). Les archives de Marius Barbeau : une richesse à découvrir ou à redécouvrir. Rabaska, 13, 218-230. https://doi.org/10.7202/1033764ar d'utilisation que vous pouvez consulter en ligne. 
(1) Jeune garçon de la famille Lemelin tenant dans ses mains le coq en métal d'une girouette, Saint-François, Île d'Orléans, Québec, 1925. Source : Musée canadien de l'histoire, photographie de Marius Barbeau no 65769.

2 Enfants autour d'un tonneau d'eau, chez Joseph Bouchard, La Misère, Les Éboulements, 1937.

Source : Musée canadien de l'histoire, photographie de Marius Barbeau nº 83580.

3 Deux benjamins d'Alphonse Dion debout à côté de leurs berceaux, Saint-François, Île d'Orléans, 1925.

Source : Musée canadien de l'histoire, photographie de Marius Barbeau nº 65734.

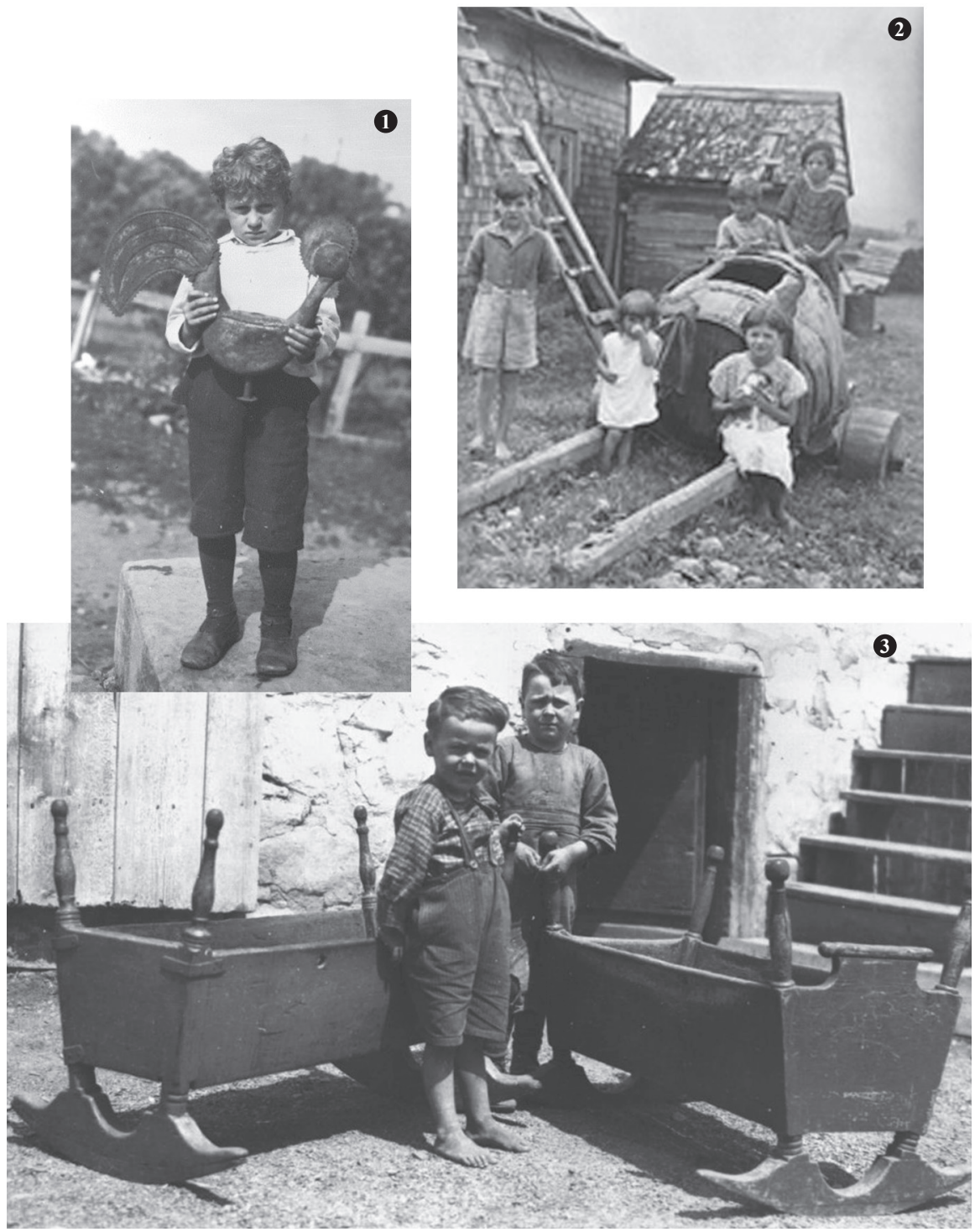




\title{
Les archives de Marius Barbeau : une richesse à découvrir ou à redécouvrir
}

BENOÎT THÉRIAULT

Musée canadien de l'histoire

\begin{abstract}
"Est-ce que tout ce travail sera perdu jusqu'à ce qu'on le retrouve, dans cinquante ans? "
\end{abstract}

Marius Barbeau

En 1965, dans une entrevue qu'il accordait à Laurence Nowry, son futur biographe, Marius Barbeau s'inquiétait que ses documents inédits puissent tomber dans l'oubli avant qu'on ne les redécouvre longtemps après son décès ${ }^{1}$. À l'évidence, les inquiétudes exprimées par Barbeau, concernant la pérennité de son travail, ne s'avérèrent pas fondées. Au Musée canadien de l'histoire, l'intérêt des chercheurs, pour le travail de Barbeau, ne s'est jamais démenti, du moins depuis les vingt dernières années. Ce qui a varié, ce sont évidemment les questionnements et la manière d'interroger les sources documentaires. Marius Barbeau lui-même et son époque sont depuis plusieurs années devenus sujets d'études ${ }^{2}$. Mais peut-on dire pour autant que les travaux de Barbeau demeurent pertinents ? Si oui, dans quelle mesure le sont-ils ?

Marius Barbeau a été l'initiateur et il a œuvré dans tant de champs de recherche différents, qui sont aujourd'hui autant de domaines spécialisés, qu'il est difficile de croire qu'il ait pu tous les maîtriser. Chose certaine dans la plupart des cas, il a agi en véritable pionnier, nous laissant, pour en témoigner,

1. «When I retire for good, well who will know where these are in my files, who will care to see, if in my own existence when I urge to get them published, and I'm interested and I'm pushing. Who will? This means that this work will be lost until it is rediscovered fifty years from now? ». Laurence Nowry, « Dr. Marius Barbeau - Canada's Pioneer Folklorist », entrevue radiophonique présentée en décembre 1965 et octobre 1966 à la CBC. Transcription par Renée Landry, Musée national de l'homme, 1982, p. 75. Musée canadien de l'histoire [MсH], Archives, « Exposition : Je suis un pionnier - Marius Barbeau (1883-1969)» (2002-I0030), boîte I-222 f.4.

2. Soulignons à titre d'exemple la publication en 2008 du collectif « Around and About Marius Barbeau » : Lynda Jessup et al., Around and About Marius Barbeau : Modelling Twentieth-Century Culture, Gatineau, Canadian Museum of Civilization, " Mercuy series, Cultural studies paper » 83, $2008,367 \mathrm{p}$. 
une abondante documentation, dont plus de 900 titres de publications, principalement des articles de journaux, de magazines et de revues spécialisés, mais aussi des monographies savantes, des recueils de chansons et de contes, des livres grand public et des écrits touristiques, des livres pour enfants et même des œuvres littéraires ${ }^{3}$. Mais peut-être, plus important encore, il nous a légué d'impressionnantes archives textuelles, photographiques et audiovisuelles qui constituent, au Musée canadien de l'histoire, le fonds Marius Barbeau.

Matériellement, le fonds Marius Barbeau se compose de plus de 600 boîtes de documents textuels (72 mètres linéaires) regroupant des carnets et notes de terrain; des dossiers de recherche ; un imposant corpus de transcriptions textuelles et musicales de chansons et de contes traditionnels; des manuscrits d'ouvrages publiés et inédits; de la correspondance professionnelle ; des documents administratifs et des copies d'articles et d'ouvrages publiés. À cela, il faut ajouter près de 15000 photographies originales de terrain, de même plus de 1500 cylindres de cire comportant 4585 chansons françaises (dont 1986 recueillis par des collaborateurs) et 3000 chants autochtones. Pour ce qui est de sa structure, le fonds Barbeau peut se diviser en trois grandes parties : les documents de terrain et dossiers de recherche ; les documents liés à la diffusion; et les documents administratifs et personnels.

D'une manière simplifiée, on peut dire que Marius Barbeau a mené de front deux carrières : celle de l'anthropologue étudiant les cultures autochtones, travail pour lequel il avait été formé à Oxford et à Paris ; puis celle du folkloriste œuvrant dans un champ d'études qu'il a lui-même élaboré et développé, souvent à l'encontre du mandat du Musée et des directives de ses supérieurs. Par intérêt de recherche et sans doute aussi par nécessité, Barbeau a toujours cherché à intégrer ces deux aspects de son travail d'ethnologue. Ceci se reflète dans sa méthodologie, mais aussi dans une certaine mesure

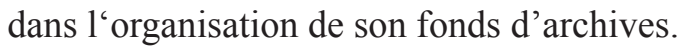

\section{I - Documents liés à la recherche \\ A. Études amérindiennes}

Cette imposante collection compte 69 boîtes de documents, soit six mètres linéaires de documents textuels auxquels s'ajoutent 3000 enregistrements sonores et des milliers de photographies. Il s'agit d'une documentation unique de première main, couvrant les divers aspects des sociétés et cultures autochtones du nord de la Colombie-Britannique d'une part et des Hurons Wyandot

3. Comme le souligne Laurence Nowry dans sa biographie de Marius Barbeau, cette production phénoménale, qui déjà stupéfiait ses contemporains, doit être relativisée. "Barbeau's by-line appears over 900 times. Much of his material appeared not merely more than once, but repeatedly. [...] Perhaps a twelfth of the titles qualify in length or content as Principal Works ». Laurence Nowry, Marius Barbeau: Man of Mana, Toronto, NC Press, 1995, p. 404. 
d'autre part : organisation sociale (clans, hiérarchie sociale), histoires de familles, cérémonies comme celle du potlatch, mythologie, linguistique, chansons, technologie, médecine, ethnobotanique, alimentation, pêche, chasse, art. Cette documentation fait toujours autorité en études autochtones et demeure l'une des plus sollicitées du fonds Barbeau, que ce soit par des chercheurs spécialisés, des étudiants, des groupes autochtones et même des firmes d'avocats. Elle est, pour les Hurons de Wendake au Québec et les Wyandottes d'Oklahoma, à la base des efforts de revitalisation de leurs traditions et de leur langue. Aussi, en 2012, ont-ils tenu à souligner le centenaire des travaux de Barbeau, en organisant une conférence internationale à Wendake et, en Oklahoma, une exposition spéciale sur le travail de ce dernier ${ }^{4}$.

\section{B. Études folkloriques (principalement au Canada français) \\ 1. Les traditions orales}

Les traditions orales, représentent le premier grand pan des recherches en études folkloriques amorcées par Barbeau en 1914. Que ce soient les chansons, la musique, la danse, les contes et les légendes, les anecdotes, les formules, les dictons, les personnages légendaires, les sobriquets, la toponymie locale ou les parlers régionaux, rien ne lui échappe. Mais c'est principalement vers les chansons, la musique, la danse et les contes que se portent tout d'abord ses efforts.

\section{Chansons, musique et danse}

Concernant la chanson traditionnelle, la musique et la danse, les archives de Marius Barbeau se divisent en deux parties.

Ses dossiers de recherche : un ensemble de documents (19 boîtes : 2,28 m) incluant analyses, comparaisons des versions canadiennes et françaises, classification par types et par titres, chansons harmonisées par Barbeau luimême, mais aussi par des musiciens reconnus tels Wilfrid Pelletier, Ernest Macmillan et plusieurs autres.

Les collections à proprement parler : a) collections d'enregistrements sonores : 4656 chansons et pièces de musique enregistrées sur cylindres de cire par Barbeau et ses collaborateurs, principalement Édouard-Zotique Massicotte et Adélard Lambert et dans une moindre mesure Jules Tremblay, Gustave Lanctôt et Loraine Wyman ${ }^{5}$; b) les transcriptions textuelles et musi-

4. Du 13 au 16 juin 2012, Congrès d'études wendat et wyandot / Wendat and Wyandot Studies Conference, organisé par le Conseil de la Nation huronne-wendat de Wendake et le Centre interuniversitaire d'études et de recherches autochtones (CÉRA) de l'Université Laval ; du 6 septembre au 25 novembre 2012, A Centennial Celebration of Dr. Charles Marius Barbeau in Oklahoma, une exposition spéciale du Sam Noble Oklahoma Museum of Natural History de Norman, Oklahoma.

5. Ceci exclut les collections sur cylindres de cire de Carmen Roy et de Marcel Rioux, qui ne font pas, à proprement parler, partie de la collection Barbeau. 
cales de ces mêmes enregistrements sur cylindres de cire (29 boîtes ou 3,5 m); c) les collections de chansons manuscrites recueillies à la dictée, soit 1915 autres pièces (13 boîtes : 1,6 m.). Le tout composant un total de 6571 chansons.

La collection de chansons traditionnelles est incontestablement celle pour laquelle Barbeau est le plus reconnu. Sa contribution dans ce domaine est essentielle. Les recueils qu'il a publiés et tous ceux qui ont découlé de ses collections ont sûrement contribué à cette renommée 6 . On peut penser, devant tout ce qui a été publié, que l'on a fait le tour de la question. Pourtant, plus que jamais, ce formidable corpus de chansons traditionnelles nous offre de nouvelles avenues de recherche. Il s'agit là d'un matériel de première main, sans le filtre des textes critiques ou des versions esthétiques qu' on peut retrouver dans les publications. Les enregistrements sur cylindres de cire, malgré leurs âpretés, nous ramènent aux documents d'origine, permettant, de ce fait, de nouvelles analyses : mode d'interprétation, prononciation, accents régionaux, etc. C'est également un instantané de la langue parlée d'il y a 70 à 100 ans, ce qui, considérant l'âge des informateurs peut nous ramener au milieu du XIX ${ }^{\mathrm{e}}$ siècle'. D'une même manière, les transcriptions textuelles et musicales sont, malgré leurs difficultés à rendre fidèlement les chansons recueillies ${ }^{8}$, des documents de base pour l'élaboration de nouvelles études.

Ainsi, en dépoussiérant un peu ces archives, les chanteurs traditionnels, tout comme les chercheurs y trouveront leur compte, car même fixées dans des enregistrements et des écrits, les chansons traditionnelles demeurent vivantes. Que ce soit directement ou indirectement, elles continuent d'avoir un impact dans notre société. Pensons simplement à l'affirmation et à l'évolution d'une identité nationale canadienne-française puis québécoise. Le mode de transmission a changé. Certains types de chansons se sont popularisés, d'autres se sont confinées dans des rôles spécifiques. Par exemple, les chansons enfantines n'ont jamais perdu la cote. Elles jouent un rôle essentiel dans le dévelop-

6. Mentionnons à titre d'exemple son Romancero du Canada publié en 1937, Alouette! (1946) et Le rossignol y chante (1962), première partie d'une trilogie devant comprendre également En roulant ma boule (1982, posthume) et Le roi boit (1987, posthume).

7. Ainsi au nombre de ceux-ci, Édouard Hovington de Tadoussac rencontré par Barbeau en 1916 était alors âgé de 85 ans. $\mathrm{M}^{\text {me }}$ Élizabeth Tremblay des Éboulements avait pour sa part 80 ans et Louis Simard (dit l'Aveugle) des Mille-Vaches était âgé de 65 ans. De l'autre côté du Saint-Laurent, en 1918, François Saint-Laurent de Sainte-Anne des Monts avait 49 ans, Ovide Soucy de Saint Antonin, 65 ans, et Luc April et $\mathrm{M}^{\mathrm{me}}$ Luc April (née Émilienne Bouchard) de Notre-Dame-du-Portage, tous deux 57 ans.

8. Cette question était particulièrement préoccupante du temps de Marius Barbeau, alors que les cylindres de cire ne permettaient que de deux à quatre minutes d'enregistrement continu. Barbeau, pour sa part, contournait ce problème en prenant à la sténographie, le plus fidèlement possible, les paroles des chansons, puis en enregistrant sur cylindre la mélodie, quelques couplets et le refrain étant suffisants. Restait ensuite le travail harassant de transcrire la musique et de retranscrire en écriture courante les paroles. 
pement cognitif des enfants. Tous connaissent « Il pleut il pleut bergère $»^{9}$. Mais cette charmante bergerette n'est peut-être pas aussi inoffensive qu'il y parait. En tous cas, la bergère n'y est pas toujours commode, comme en fait foi une version de cette chanson enregistrée en 1916 auprès de $\mathrm{M}^{\text {lle }}$ Adèle Lamothe, et dont voici un extrait :

Permet qu'à ton corsage j'attache ce ruban

Qu'il te soit le doux gage de tous mes sentiments

Tout ça, monsieur m'honore, Mais moë, j'ai mes sabots

Si vous m' touchez encore, Je vous les casserai su'l dos. ${ }^{10}$

Voilà qui a le mérite d'être clair. À ce propos, les bergères sont assez rares au Québec. Elles sont plus présentes dans les chansons que dans les prés. Chez Barbeau, elles sont plus de 350, toutes immigrantes, je suppose. Toutefois, comme le souligne Conrad Laforte, il ne faut pas s'y méprendre, car, dans la chanson d'origine médiévale, la bergère désignait simplement une amoureuse ${ }^{11}$.

\section{Contes et légendes}

Tout aussi riche que celle des chansons, la collection des contes et légendes représente à elle seule dix boîtes de documents textuels $(1,2 \mathrm{~m})$. Objet de ses premiers terrains en 1914, les contes et les légendes ont été également largement diffusés par Barbeau et par tous ceux qui, après lui, s'y sont intéressés. Malgré les difficultés inhérentes à leurs collectes, Barbeau a toujours cherché à recueillir les contes de la manière la plus fidèle possible. Ce qui en fait, encore aujourd'hui, des sources fiables. Toutefois, malgré le retour en force, ces dernières années, de l'intérêt pour les contes et les légendes, il semble y avoir une cassure entre les praticiens actuels et le conte traditionnel. Pourtant, les exploits de l'éternel Ti-Jean n'auraient-ils pas, encore aujourd'hui, de quoi inspirer?

\section{Arts, métiers traditionnels et savoir-faire}

L'étude des arts, des métiers traditionnels et des savoir-faire est l'autre grand pan des recherches de Marius Barbeau. Il s'y est consacré pleinement et avec passion tout au long de sa carrière. Curieusement, les travaux de Barbeau dans ces domaines sont généralement méconnus. Pourtant, que ce soient les arts (orfèvrerie, sculpture, peinture), les arts domestiques, les métiers tradition-

9. Écrite en 1780 par Fabre d'Églantine pour l'opérette Laure et Pétrarque, cette chanson, en intégrant le répertoire de la chanson traditionnelle, s'en trouvera à l'évidence « enrichie ».

10. Adèle Lamothe, Il pleut, il pleut bergère, Montréal, 1916, chanson MN 2. McH, Archives, Fonds Marius Barbeau, «Transcriptions textuelles et musicales », boîte B114 f.2.

11. Conrad Laforte, "La Chanson de tradition orale, patrimoine poétique et musical », Les Cahiers de l'Association pour l'avancement de la recherche en musique au Québec, 1995, nº 16, p. 59. 
nels (charpentiers de navires, menuisiers, couvreurs, forgerons, ferblantiers, cordonniers, cordiers, potiers, horlogers, etc.), l'architecture classique et domestique, l'agriculture et les pêches, Barbeau fut l'un des premiers à s'y intéresser. Dans tous ces domaines, il fit œuvre de précurseur. Sur tous ces sujets, il nous a laissé de généreuses notes d'enquêtes, des dossiers de recherche copieux regroupant des documents de toutes sources, de la correspondance, des compilations de sources archivistiques, et de multiples photographies de terrain destinés à documenter chacun de ses sujets de recherche. Ce matériel, pour donner un ordre de grandeur, représente 32 boîtes de documents (près de 4 mètres linéaires).

Les recherches menées par Barbeau en orfèvrerie, en sculpture et en peinture anciennes ( $\mathrm{du} \mathrm{XVII}{ }^{\mathrm{e}}$ au milieu du XIX ${ }^{\mathrm{e}}$ siècle), sont particulièrement remarquables par leur richesse documentaire, résultat de patientes prospections, entres autres, dans les archives des communautés religieuses et dans les anciennes paroisses du Québec. En orfèvrerie de la Nouvelle-France, on ne pense pas spontanément aux travaux de Barbeau; mais bien davantage à ceux de Gérard Morisset et à son inventaire des œuvres d'art du Québec, lancé en 1937. Toutefois, bien que les publications de Barbeau sur l'orfèvrerie n'aient pas l'ampleur de celles de Morisset, ses documents de recherche n'en sont pas moins importants. En fait, les recherches de Barbeau, qui ont débuté en 1925, sont généralement antérieures à celles de Morisset, ce qui leur confère un intérêt supplémentaire.

Ses travaux sur la sculpture et la peinture, conduits en parallèle avec ses recherches sur l'orfèvrerie, sont sensiblement du même ordre. Mais ici, le travail de Barbeau se fait à deux niveaux. Premièrement, il documente les œuvres et les artistes ayant œuvré du XVII ${ }^{\mathrm{e}}$ au XIX ${ }^{\mathrm{e}}$ siècle auxquels il ajoute, notamment chez les sculpteurs, des artistes contemporains tels Louis Jobin et dans une certaine mesure son protégé Médard Bourgault, chez qui il voyait des influences de l'école de Quévillon.

Ses oncles étaient menuisiers-sculpteurs, entre autres l'oncle Legros, de qui il tient son penchant pour la sculpture. Sa paroisse était naguère un centre d'artisans pour la construction et la décoration des églises, dans tout le bas du fleuve. Les premiers maîtres sculpteurs, Perrault et Charron, qui vinrent s'y établir, il y a plus de cent ans, étaient de l'école de l'Île-Jésus et ils pratiquaient le style de Quevillon. ${ }^{12}$

12. Marius Barbeau, "Charité bien ordonnée », La Presse, Montréal, samedi 3 juin 1933, vol. 49, $\mathrm{n}^{\circ}$ 193. p. 45. Мсн, Archives, Fonds Marius Barbeau, « Coupures de presse », B-Mp-228_1, boîte B152. Cette allusion à l'école de Quévillon, Barbeau la reprendra entre autres dans ses cours à l'Université Laval : « Bourgault - autodidactes, mais se rattachent à Chisostome Perrault et Amable Charron (Quévilon) ». (cf. « Nos arts populaires - rustiques », 7 juillet 1943. Conférences et cours - Université Laval - Québec été 1943. Mch, Archives, Fonds Marius Barbeau, boîte B356 f5). 
Puis il se fait le promoteur et parfois l'agent d'artistes contemporains, écrivant des articles à leurs sujets, utilisant leurs œuvres pour illustrer ses articles et ses livres, intégrant leurs œuvres dans des expositions, encourageant ses relations et les musées à acquérir des œuvres, etc. Ce sera le cas pour le sculpteur Médard Bourgault, à qui, en début de carrière, il donnera même des conseils sur la réalisation de ses œuvres, l'incitant du même souffle à se distancier du travail de son frère dont il juge les petites statuettes « pas encore vraiment intéressantes » et pouvant, si on les confond aux sculptures de celui-ci, nuire à son $«$ commerce $»^{13}$.

Certaines de vos choses $[\ldots]$ sont à peu près parfaites. D'autres pourraient être rendues meilleures par de la simplification, comme le grand crucifix qui a beaucoup trop de muscles et de nerf, etc. En art, il faut simplifier et souvent s'en tenir aux grands plans. [...] il ne faut pas trop rider les visages des personnages. Tenez compte de ceci, que la simplification des détails augmente l'énergie de l'ensemble. Ces remarques pourront vous être utiles. ${ }^{14}$

Il encourage de même Emily Carr, les peintres du Groupe des Sept, dont ses amis A.Y [Alexander Young] Jackson et Arthur Lismer, ou encore Langdon Kihn et Henri Masson. En fait, Barbeau est ami ou en relation avec près de cinquante peintres contemporains, dont les surréalistes Wolfgang Paalen et Kurt Seligmann. Il aide également de jeunes artistes dont le peintre Jean Dallaire pour qui il trouve un mécène pouvant lui payer ses études à l'École des beaux-arts de Montréal, ce qui lui permettra en octobre 1938 d'obtenir une bourse pour étudier à Paris. Malheureusement, il y était toujours lors de l'entrée en guerre du Canada en 1939 et sera captif durant toute la guerre ${ }^{15}$.

Dans le domaine des arts domestiques, Barbeau n'agit pas différemment. Il documente œuvres et techniques, il s'intéresse à tout : costume, art du tissage, boutonné, broderie, tapis crocheté, ceinture fléchée, mais aussi ouvrages anciens, des communautés religieuses notamment qu'il surnomme les saintes artisanes.

Ici également, les archives de Barbeau témoignent de ces deux niveaux d'intérêt. Le travail de terrain et l'acquisition de pièces intéressantes pour le Musée ; puis la promotion de toutes les manières possibles de cet art qui le passionne. Il se fait l'agent des artisanes. Il incite ses amis à se procurer des pièces anciennes, mais aussi à passer des commandes, il fait acquérir des pièces par toutes les institutions possibles : Galerie nationale du Canada, Musée royal de l'Ontario, Musée du Fort-Chambly, Maison Laurier. Il persuade des hôtels à faire confectionner des textiles, des couvertures et des tapis

13. Lettre de Marius Barbeau à Médard Bourgault, 29 juin 1933. МсH, Archives, Correspondance de Marius Barbeau « Bourgault, Médard (1930-1953) », boîte B174 f.26.

14. Lettre de Marius Barbeau à Médard Bourgault, 11 avril 1935. Ibid.

15. МсH, Archives, Correspondance de Marius Barbeau, « Dallaire, Jean-Philippe (1938, 1943, 1953) », boîte B185 f.9. 
pour des chambres canadiennes. Il encourage les tisserandes, leur obtient des contrats, garde des pièces en consigne et donne même son avis sur ce que devraient être des pièces traditionnelles. Par exemple, le 15 avril 1926, il écrit à Madame F.X. Cimon, Baie-Saint-Paul :

[...] Je suis heureux d'apprendre de Miss Kate Treleaven, Office of the President, C.P.R., Montréal, de la part de M. Beaty lui-même qu'ils ont été si enchantés de votre travail qu'ils veulent vous demander un certain nombre de pièces, comme suit : Bleu avec nervures 24 verges ; Rose avec nervures 30 verges; Droguet bleu (bleuâtre) et rose 14 verges ; orange veiné 15 verges ; vert avec nervures 37 verges ; orange (brins doubles) 33 verges ; beige-orange 16 verges ; jaune et vert et orange 10 verges; vert (brins doubles) 6 verges [...]. J'ai mentionné à Miss Treleaven par écrit que le prix mentionné était de $\$ 4.00$ la verge [...]. Je suis heureux d'obtenir pour vous cette commande, surtout si cela vous fait plaisir et à vos belles demoiselles. ${ }^{16}$

Autre exemple, ce mot adressé le 13 juillet 1933 à Madame Germain H. Demeules, La Baleine, Isle-aux-Coudres :

Chère Mme Demeules, $\mathrm{Ci}$ - inclus vous trouverez un chèque pour $\$ 14.70$, qui couvre le tapis envoyé à l'Alberta $(\$ 10.40)$, et la couverture de flanelle épaisse vert et rouge (\$4.30). Il reste encore un petit tapis du castor qui n'est pas vendu. Dans votre dernière lettre vous m'annoncez l'envoi de plusieurs couvertures. Je ne les ai pas encore reçues, mais je les attends. ${ }^{17}$

Au niveau des savoir-faire, s'il est une chose qui n'a pas échappé à Barbeau, comme marqueur identitaire, c'est bien la cuisine traditionnelle. Bien que singulière par son adaptation au terroir, elle ne pouvait sans doute que refléter ses origines françaises. L'attrait touristique de cette spécificité gastronomique n'échappait pas non plus à Barbeau qui, en 1927 lors du festival de folklore de Québec, réussit à convaincre les directeurs du Château Frontenac d'offrir à leur clientèle un menu typiquement « canadien-français» ${ }^{18}$. À partir de 1936, Barbeau poussant plus avant ses recherches se mettra en quête de

16. Lettre de Marius Barbeau à Alice (Tremblay) Cimon, 15 avril 1926. Mch, Archives, Correspondance de Marius Barbeau « Cimon, Alice (née Tremblay) (1926-1927) », boîte B181 f.9.

17. Lettre de Marius Barbeau à $\mathrm{M}^{\mathrm{me}}$ Germain Hilaire Demeules, 3 juillet 1933. Мсн, Archives, Correspondance de Marius Barbeau « Demeules, Germain Hilaire $\left(\mathrm{M}^{\mathrm{me}}\right),(1932-1946) »$, boîte B187 f.36.

18. En 1927, John Murray Gibbons, agent de publicité du Canadien Pacifique, avait confié à Marius Barbeau, la programmation du « Festival de la chanson et des métiers du terroir » tenu au Château Frontenac à Québec du 20 au 22 mai 1927. Désirant offrir une expérience globale aux touristes venant pour le festival, Barbeau avait eu l'idée d'un menu « canadien-français », cuisiné spécialement, le temps du festival, par quelques femmes de l'île d'Orléans. D'abord rejetée, l'idée fut finalement acceptée deux ou trois jours avant le véritable début des activités, ce qui obligea Barbeau à bien des prouesses, d'autant qu'il fallut faire venir des pois de l'île d'Orléans en catastrophe, ceux des cuisines du Château Frontenac s'étant révélés impropres pour la soupe aux pois. $C f$. «Les mémoires de Marius Barbeau » (1957-1958), p. 173-174. Мсн, Archives, Fonds Carmen Roy, « Les mémoires de Marius Barbeau » (1957-1958), (2000-F0003), boîte 624, f.10. 
la cuisine traditionnelle, recueillant en peu de temps, à l'aide de collaboratrices, un important corpus de plusieurs centaines de recettes de cuisine (36 cm linéaires de documents) ${ }^{19}$. Conservées dans le fonds Barbeau, elles s'y retrouvent classées par catégories : soupes et potages ; pâtés, cretons, ragouts et boudins ; légumes et bouillis ; gibiers et volailles ; poissons ; marinades, herbes salées et sauces ; breuvages, vins et vinaigres ; crêpes et omelettes ; recettes de laits et fromages ; desserts, fruits, tartes, pâtisseries, biscuits, brioches, galettes et poudings ; pains ; beignes et croquignoles. Tout y est, de la liqueur d'angélique au flan à l'érable en passant par la soupe aux pois, les fromages artisanaux (dont ceux de l'île d'Orléans), les langues de morue, l'oie rôtie, la tourtière, les cretons, les tartes en tout genre, et tout le reste. Un délice ! Le tout mesuré à la pincée et à l'œil évidemment. Bien que l'on puisse se demander dans quelle mesure ces recettes sont traditionnelles ou issues d'échanges interculturels, cette collection n'en demeure pas moins des plus intéressantes.

\section{II - Les documents liés à la diffusion}

Dans tout ce que fait Barbeau, la nécessité de diffuser revient comme une obsession. L'abondance et la variété de ses publications en sont la meilleure preuve. Plus de 900 titres $^{20}$, ce qui malgré les nombreuses redites, demeure phénoménal. Il y a là, déjà, plusieurs sujets d'étude possibles, notamment, sur l'impact de celles-ci sur la compréhension par le grand public de ce que sont la culture traditionnelle et le folklore. Si l'on ajoute à cela une centaine de textes ou plans de conférences et toutes les coupures de presse s'y rapportant, on obtient une documentation de grand intérêt. Mais pour Barbeau la diffusion ne passe pas que par les publications et les conférences, mais également par les concerts de chansons traditionnelles, les festivals, les expositions, les films, les émissions radiophoniques et télévisuelles, et par l'enseignement.

L'enseignement, c'est là l'aboutissement logique de sa carrière. Il nous a laissé pour en témoigner 13 boîtes de notes de cours données dans les années 1940 et 1950 aux universités d'Ottawa, Montréal et Laval à Québec ; elles font la synthèse de ses expériences, de ses conceptions de l'anthropologie, du folklore et du travail de terrain ${ }^{21}$.

19. Bien que Barbeau puise à toutes les sources, les recettes recueillies proviennent essentiellement de la région de Québec, de l'île d'Orléans, et vers l'est des deux côtés du fleuve : côte du Sud, du Bas-Saint-Laurent, Gaspésie de même que Côte-de-Beaupré, Charlevoix et Saguenay.

20. Le nombre précis des publications de Marius Barbeau demeure incertain. Mais l'on s'entend normalement à le situer entre 900 et 1000 .

21. Au sujet des cours donnés par Marius Barbeau, voir : Marius Barbeau, « Comment on recueille les contes, les chants, les mélodies, le langage, les spécimens ou les données s'y rapportant, parmi les sauvages ou les blancs ", Rabaska, Revue d'ethnologie de l'Amérique française, volume 9, 2011, p. 171-181 : «Inédit». 


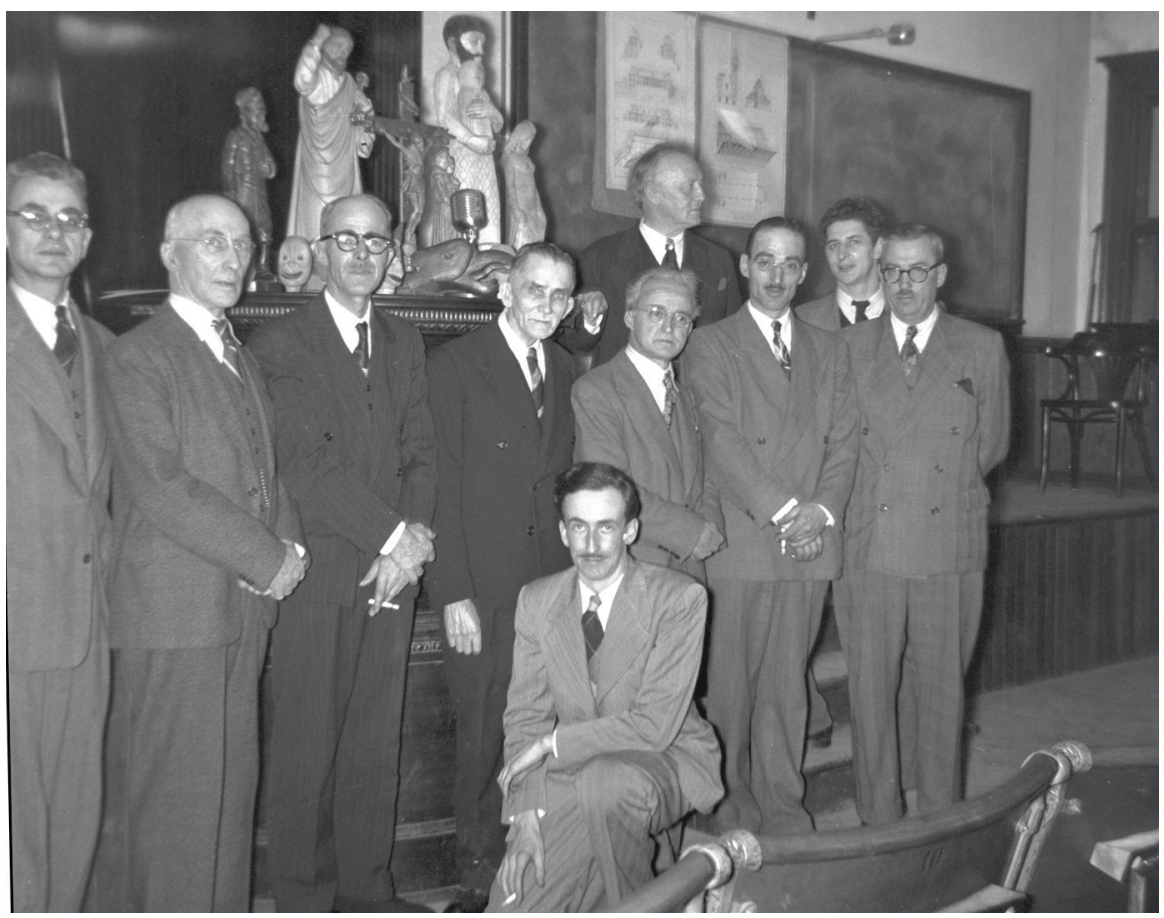

Participants à la conférence de Marius Barbeau,

« Maîtres menuisiers et sculpteurs (1646-1950) » donnée le 14 mars 1950, dans le cadre des « Mardis universitaires » de l'Université Laval.

De gauche à droite : le peintre Jean-Paul Lemieux ; l'architecte Pierre Lévesque ; les sculpteurs Médard Bourgault, Henri Angers, Lauréat Vallière et Jean-Julien

Bourgault ; Paul Rainville conservateur du Musée de la Province de Québec.

Accroupi à l'avant, on reconnaît le sculpteur Clément Paré et, debout à l'arrière à droite, Marius Barbeau et Luc Lacourcière.

Source : Musée canadien de l'histoire, photo 99-1.

\section{III - Les documents administratifs}

De toutes ses archives, les documents, qui à notre sens permettent le mieux de comprendre et de faire les liens entre tous les champs d'intérêt de Marius Barbeau, sont les documents administratifs et sa correspondance.

C'est là que se trouvent les informations sur ses terrains, sur l'acquisition d'objets, tant pour Musée national du Canada que pour plusieurs autres. On y découvre ses conceptions de ce qu'est la muséologie : quoi choisir, comment le présenter, mais surtout que considère-t-il comme étant représentatif? L'exemple de la Maison Laurier à Saint-Lin en 1939 est intéressant à ce sujet ${ }^{22}$. Barbeau, qui ne trouve pas ce qu'il cherche localement, se tourne

22. À ce sujet, le rapport préliminaire de Barbeau, remis au directeur des Parcs nationaux au début de 1939, est très éloquent. Ses conceptions de ce qui est représentatif d'une maison de village typique de l'époque de la naissance de sir Wilfrid Laurier (1841) y sont clairement exprimées. $C f$. Marius 
vers Québec, l'île d'Orléans et la côte de Beaupré pour habiller l'intérieur de la maison. Il ajoute à cela des couvertures et des boutonnés de Charlevoix et fait tapisser les murs d'une toile de lin à aigrettes ${ }^{23}$, tissés exprès par les tisserandes des Éboulements et de l'Isle-aux-Coudres, leur procurant de ce fait un contrat lucratif bien apprécié24.

Enfin, une des plus grandes richesses du fonds Barbeau, avec tout ce qu'elle peut nous apporter de sujets de recherche et de compréhension du milieu et de l'époque du chercheur, reste sans contredit sa correspondance professionnelle : soit 92 boîtes de document, comportant 5400 dossiers de correspondance inédite, de 1910 à 1968, avec des chercheurs, écrivains, artistes, artisans, gens ordinaires, étudiants, collaborateurs, élite politique et religieuse, chanteurs traditionnels, professionnels, photographes, anthropologues, folkloristes, galeristes, amateurs d'art, éditeurs, cinéastes, journalistes et bien d'autres, et ce, de tout le Canada, des États-Unis et d'Europe. Voir en annexe, à titre d'exemple, une courte sélection de noms de correspondants.

En conclusion, nous croyons que Marius Barbeau peut dormir en paix. Par ses méthodes de recherche qu'il voulait scientifiques, il a su s'assurer que ses collections et ses documents de recherche demeurent ou redeviennent pertinentes et ce même après un siècle. Que ce soit par ses documents de terrain, ses dossiers de recherche, ceux liés à la diffusion ou encore par ses documents administratifs, nous n'avons pas fini de trouver de nouvelles avenues de recherche dans l'héritage qu'il nous a laissé.

Car, si on en croit Alain Grandbois : « Les totems le protègent, il ne mourra jamais. ${ }^{25}$ »

Barbeau « Concerning the Laurier Memorial at St. Lin, P.Q. » [janvier 1939]. Mch, Archives, Fonds Marius Barbeau, « Maison Laurier, St-Lin (1939-1940) », boîte B366.

23. Il s'agit d'une grosse toile rugueuse contenant des particules d'écorce de lin.

24. Bien que représentant un revenu apprécié $\left(80 \$\right.$ pour $\mathrm{M}^{\mathrm{me}}$ Rose Dufour et ses sœurs et $45 \$$ pour $\mathrm{M}^{\text {me }}$ Aquilas Girard), ce travail demeurait hors norme par la quantité de toile demandée : 170 verges carrées (170 verges x 36 po de large). En fait même la disponibilité du lin fit problème. Si bien que Barbeau se mit lui-même à la recherche de la filasse nécessaire, qu'il trouva à la Coopérative de Vaudreuil et Soulanges. $C f$. Lettres de Marius Barbeau à $\mathrm{M}^{\text {Ile }}$ Rose Dufour, 10 décembre 1938; 17 février 1939. Мсн, Archives, Correspondance de Marius Barbeau, « Dufour, Rose (1938- 1948) », boîte B190, f.13. Marius Barbeau « Furniture, Equipment and Decoration for the Laurier Memorial House at St. Lin, Québec » [mars 1939]. Mch, Archives, Fonds Marius Barbeau, « Maison Laurier, St-Lin (1939-1940) », boîte B366.

25. Alain Grandbois « Marius Barbeau », BANQ Montréal, fonds Alain Granbois (MSS-204). Poèmes inédits, poèmes incomplets, recueils, 204/1/1 à 204/1/37. 


\section{Annexe \\ Fonds Marius Barbeau \\ Liste sélective et aléatoire de correspondants}

\section{Anthropologues :}

Robert Ranulph Marett (1911-1942) ; Marcel Mauss (1913-1926) ; Franz Boas (1914-1940).

\section{Politiciens :}

Très-Hon. Louis Saint-Laurent (1911-1962) ; Sir Wilfrid Laurier (1916) ; L'hon. Alexandre Taschereau (1917, 1931-1934) ; Très-Hon. William Lyon MacKenzie King (1939-1948); Vincent Massey (1926-1958).

\section{Musiciens et chanteurs :}

Sir Ernest MacMillan (1926-1963) ; Wilfrid Pelletier (1928-1961) ; Claude Champagne (1929-1961) ; Léo-Pol Morin (1922-1930); Émile Boucher (1937-1963); Lionel Daunais (1932, 1963); Juliette Gaultier de la Vérendrye (1925-1938); Yvette Guilbert (1916-1920); Charles Marchand (1927-1930); Oscar O’Brien (1928-1943).

\section{Sculpteurs et peintres :}

Sylvia Daoust (1942-1943) ; Louis Jobin (1925-1927) ; Lauréat Vallière (1928-1962); Charles F. Comfort (1937-1963); Marcelle Ferron (1963); Marc-Aurèle Fortin (1931-1940) ; Alex Y. Jackson (1925-1961) ; Jean-Paul Lemieux (1949-1959) ; Arthur Lismer (1925-1960) ; Alfred Pellan (1943-1945) ; Marc Aurèle De Foy Suzor-Côté, (1920-1933).

\section{Muséologues :}

Gérard Morisset (1934-1956) ; Paul Rainville (1933-1948).

\section{Collaborateurs :}

Édouard-Zotique Massicotte (1917-1947) ; Père Archange Godbout (1917-1961) ; Adélard Lambert (1919-1957); Abbé Victor Tremblay (1937-1962); Joseph Thomas Le Blanc (1939-1940) ; François-J. Brassard (1940-1960); M ${ }^{\mathrm{gr}}$ Félix Antoine Savard (1942-1958) ; Luc Lacourcière (1939-1962). 